\title{
Atrial Fibrillation and Cardio-embolic Stroke, Incidence of Haemorrhagic Transformation and Rehabilitation Outcomes
}

\author{
S.D Pande*, Lolong L, Kamal A, Kyaw K, Kam C and Thiri M \\ Consultant Rehabilitation medicine, Changi General Hospital, Singapore
}

Received: September 01, 2015; Accepted: October 06, 2015; Published: October 09, 2015

*Corresponding author: S.D Pande, Consultant Rehabilitation Medicine, Changi General Hospital, 2 Simei St 3, Singapore, 529889, Tel:+ 6591149559; E-mail: shrikantpande@yahoo.co.uk

\begin{abstract}
Atrial Fibrillation (AF) is the most common cardiac arrhythmia. The prevalence in general population is about $0.4 \%$ and it increases with age. Stroke secondary to AF have high morbidity and mortality. We discuss the retrospective analysis of cardio-embolic strokes, in relation to the incidence of haemorrhagic transformation and its relation to $\mathrm{AF}$ along-with rehabilitation outcomes and mortality.

52 patient's data with diagnosis of cardio-embolic stroke were reviewed. 75\% (39) had diagnosis of AF before or on admission. Of all the patients, $82 \%$ (43) had diagnosis of HT. Within the HT group of patients, $67 \%$ (29) had underlying AF and all 9 patients with ischaemic stroke had underlying AF.

Only 3 patients received rt-PA and 61\% (32) were taking antiplatelet or anticoagulant therapy at the time of admission. We found a high incidence of haemorrhagic conversion in stroke patients with $\mathrm{AF}$, without thrombolysis, with some association with advancing age and possible antiplatelets.
\end{abstract}

In our limited study group high mortality (32\%) was associated with ischaemic stroke with underlying AF.

Keywords: Atrial Fibrillation (AF); Stroke; Transient Ischaemic Attacks (TIA); Hypertension (HTN); Non-Insulin-Dependent Diabetes Mellitus (NIDDM); Ischaemic Heart Disease (IHD); Functional Independence Measure (FIM); Congestive Cardiac Failure (CCF); Recombinant Tissue Plasminogen Activator (rtPA); Hemorrhagic Transformation (HT); Hemorrhagic Infarction (HI); Parenchymal Hemorrhage (PH); Symptomatic Intracranial Hemorrhage (SICH); Second European-Australasian Acute Stroke Study (ECASS II)

\section{Introduction}

Atrial Fibrillation (AF) is the most common cardiac arrhythmia. The prevalence in general population is about 0.4 $\%$ and increase with age. AF disturbs synchronous mechanical activity and impairs haemodynamics, which may lead to thrombus formation, mostly in the left atrial appendage and lead to systemic embolization. Clinical manifestations are often neurological such as transient ischaemic attacks or ischaemic strokes. One in every 6 strokes occurs in patients with AF. Antiarrhythmic therapy is important to prevent $\mathrm{AF}$ in patients with paroxysmal forms, however, to reduce the risk of emboli, antithrombotic therapy is paramount importance. The therapeutic dose of warfarin or modern anticoagulants reduces the risk of first or recurrent strokes by about $80 \%$. Aspirin is comparatively less effective (20\%) [1,2].

Intravenous thrombolysis with Recombinant Tissue Plasminogen Activator (rtPA) improves the outcome for ischemic stroke patients who can be treated within 3 hours of symptom onset. The efficacy of thrombolysis has been demonstrated despite an increased risk of severe Hemorrhagic Transformation (HT) in patients treated with rtPA [3].

Anticoagulation remains treatment of choice for AF with high or intermediate risk i.e. history of Transient Ischaemic Attacks (TIA), Stroke, age 65 and above, Hypertension (HTN), Non-Insulin-Dependent Diabetes Mellitus (NIDDM), Congestive Cardiac Failure (CCF), structural heart disease, systolic dysfunction [1].

\section{Aim}

To review the incidence of haemorrhagic transformation and rehabilitation outcomes in persons with the diagnosis of cardio embolic stroke with underlying atrial fibrillation.

\section{Methods}

This is the retrospective study of 52 consecutive patients admitted with the diagnosis of cardio-embolic stroke. These were transferred to in-patient rehabilitation center with comprehensive and multidisciplinary rehabilitation services were given in a tertiary hospital of Singapore.

The data collected was: demographic details, co-morbidity, investigations results (CT, MRI, 12 leads ECG and 24 hours Holter ECG, Echocardiogram) to confirm the diagnosis and co-morbidity, and pre-admissions medical lists were collected.

Functional Independence Measure (FIM) reports done by team members who were FIM accredited, on admission and discharges were used to measure the dependency. Mortality over 
the period of 1 to 6 years was reviewed and analyzed between two groups who survived between this period and who were not.

\section{Results}

All the patients in the study group had the diagnosis of cardioembolic stroke, confirmed clinically with neurological deficit, CT and or MRI findings.

Out of initial 60, 8 patient's data was excluded due to insufficient or inaccurate details.

Of the remaining 52, Male to female ratio was $26: 26.39(75 \%)$ had AF confirmed prior to or on admission. Of all the patients, $82 \%$ (43) had diagnosis of HT.

Within the HT group of patients, 55\% (29) had underlying AF and all 9 patients with ischaemic stroke had underlying AF.

Of all the data reviewed only 3 patients received rt-PA, and 32 $(61 \%)$ patients were taking antiplatelet or anticoagulant therapy until the diagnosis of stroke.

Thrombolytic treatment was not given, either because of the time window to hospital, presence of haemorrhage on first scan, family's refusal of treatment or other contraindications.

For the purpose of analysis, we divided patients into survivor (group 1) and non-survivor (group 2) groups.

\section{Study Group 1}

Out of the total 35 patients, 17 were males vs. 18 females with the median age of 66.7. 65\% (23) had the diagnosis of AF before or on admission.

32 of 35(91\%) had HT, 3 had ischemic stroke.

\section{Study Group 2}

17 patients died, of which 9 were male and 8 were females, with the median age of 74.8. $11(65 \%)$ had HT and 6(35\%) had ischaemic stroke.

Mean time since stroke and death was 1.04 years (IQR 0.88 to 3 years).

The distribution of stroke territories and co-morbidities are summarized in the Table 1.

Rehabilitation outcomes: the FIM scores average in (Group 1) 64.4 vs. 60.9(Group 2). For the survivors of cardio-embolic stroke patients the overall morbidity was higher, disability scores (FIM) and dependency level was higher.

The length of inpatient stay was (group 1) 25 days vs. 30 days (group 2) as compared to our overall average length of stay in the rehabilitation department (16 days).

When we compared group 1 vs. group 2(for mortality), the average age was 6.9 years higher (age 66.7 vs. 74.8 ).

In the univariate model: age, hyperlipidemia were statistically significant factors. With every one-year increase in age, there was $7.5 \%$ increase in the odds of having death as an outcome. (OR $95 \%$, CI $0.9 \%-14.5 \%, p=0.03$ )
Table 1: Patient variables in both groups.

\begin{tabular}{|c|c|c|}
\hline & Group 1 (Survivor) & $\begin{array}{l}\text { Group } 2 \text { (Non- } \\
\text { Survivor) }\end{array}$ \\
\hline Number of Males & 17 & 9 \\
\hline Number of Females & 18 & 8 \\
\hline Age Range & $41-88$ & $49-84$ \\
\hline Average Age & 66.7 & 74.8 \\
\hline $\begin{array}{c}\text { Distribution of stroke } \\
\text { territories: }\end{array}$ & & - \\
\hline $\begin{array}{c}\text { Right MCA territory infarct } \\
\text { with HT }\end{array}$ & 16 & 5 \\
\hline Left MCA infarct with HT & 13 & 4 \\
\hline $\begin{array}{l}\text { Posterior circulation infarct } \\
\text { with HT }\end{array}$ & 3 & 2 \\
\hline Ischaemic infarct & 3 & 6 \\
\hline Commonest co-morbidities: & $\begin{array}{c}\text { HTN: } 24 \\
\text { NIDDM: } 14 \\
\text { IHD: } 14 \\
\text { CCF } 6(2 \text { with dilated } \\
\text { cardiomyopathy) } \\
\text { Chronic renal } \\
\text { impairment: } 2 \\
\text { Rheumatic valve } \\
\text { disease: } 1\end{array}$ & $\begin{array}{c}\text { HTN: } 12 \\
\text { NIDDM: } 10 \\
\text { IHD: } 8 \\
\text { CCF: } 3 \\
\text { Hepatic } \\
\text { cirrhosis: } 1 \\
\text { End stage renal } \\
\text { failure: } 2\end{array}$ \\
\hline $\begin{array}{l}\text { Premorbid antiplatelet or } \\
\text { anticoagulation therapy }\end{array}$ & $\begin{array}{c}\text { Total: } 25 \\
\text { Aspirin or Clopidogrel } \\
\text { and or Dipyridamole: } \\
20 \\
\text { Warfarin: } 2 \\
\text { 3 Received rt-PA } 3\end{array}$ & $\begin{array}{c}\text { Total: } 10 \\
\text { Aspirin: } 7 \\
\text { Warfarin: } 3 \\
\text { None had rt-PA }\end{array}$ \\
\hline $\begin{array}{l}\text { Median time between stroke } \\
\text { and death }\end{array}$ & - & 1.04 years \\
\hline
\end{tabular}

MCA: Middle Cerebral Artery; HC: Haemorrhagic Conversion; HTN: Hypertension; NIDDM: Non-Insulin-Dependent Diabetes Mellitus; IHD: Ischaemic Heart Disease; CCF: Congestive Cardiac Failure; rtPA: recombinant tissue Plasminogen Activator

Among all the co-morbidities, patients with hyperlipidemia were less likely to die. (OR 0.1, 95\% CI, 0.1-0.9, $p=0.03$ ).

Patients with haemorrhagic transformation were less likely to die (OR 0.1, 95\% CI 0.1-0.5, $p=0.01$ ).

However, these factors were no more significant in logistic regression model. Diabetics were more likely to die (OR 95\% CI 1.4-708.4, $p=0.03$ )

Statistical analysis failed to support any association between preadmission AF $(p=1.00)$, antiplatelet $(p=0.32) /$ anticoagulant $(p=1.00)$ therapy until the day of stroke with HT.

\section{Discussion}

The impact of non-rheumatic AF, HTN, coronary heart disease, and CCF on stroke incidence was examined in the Framingham Study after 34 years of follow-up. Compared with subjects free of these conditions, the age-adjusted incidence of stroke was more than doubled in the presence of coronary heart disease and more than tripled in the presence of hypertension. There was more 
than a fourfold excess of stroke in subjects with cardiac failure and a near fivefold excess when atrial fibrillation was present [4].

The attributable risk of stroke for all cardiovascular contributors decreased with age except for atrial fibrillation, for which the attributable risk increased significantly, rising from $1.5 \%$ for those aged $50-59$ years to $23.5 \%$ for those, aged 80-89 years. While these findings highlight the impact of each cardiovascular condition on the risk of stroke, the data suggest that the elderly are particularly vulnerable to stroke when atrial fibrillation is present [4].

In the Framingham study, AF was associated with increased stroke severity. Thirty-day mortality was greater in AF strokes than in non-AF strokes [5].

Ischemic stroke associated with $\mathrm{AF}$ was nearly twice as likely to be fatal as non-AF stroke. Recurrence was more frequent, and functional deficits were more likely to be severe among survivors [3].

In our limited sample of 52 subjects with AF with stroke, only $18 \%$ had ischaemic strokes $(9 / 52), 43(82 \%)$ had HT; the mortality was $32 \%$ with the median time of 1.04 years since stroke.

Out of 17 patients who died, 11 had HT and 6 had ischaemic strokes. All the ischaemic stroke patients had premorbid confirmed diagnosis of AF. These findings reiterate the high mortality associated with Stroke and underlying AF.

Several hypotheses of pathogenesis are of interest with respect to the association of $\mathrm{AF}$ with worsened stroke outcome. Some authors speculated that, the higher prevalence of ischemic heart disease and congestive heart failure in AF subjects would have contributed to a reduced cerebral blood flow when the cerebral autoregulatory mechanisms are impaired as in an ischemic region $[4,5]$.

In our study, total numbers of patients with IHD were 22 and CCF was 9. But the statistical analysis failed to confirm any strong association with overall HT or mortality.

AF itself seemed to carry an excess risk of early mortality and poor functional outcome independent of the underlying cardiac disease. Other researchers have found that chronic AF may cause a significant reduction in regional cerebral blood flow [4,5].

In terms of the stroke mechanism, collateral circulation in the brain may be less developed in patients with a sudden interruption of blood flow caused by an embolus than in those who suffer a stroke as the result of an underlying chronic arterial atherosclerotic disease. These two factors may further compromise the cerebral circulation, increase the infarction size, and retard the recovery of function after ischemia associated with AF $[4,5]$.

Secondary analysis of ECASS II has confirmed the importance of the extent of hypoattenuation as a risk factor for severe HT. The findings also suggested that older patients and those who have used aspirin before stroke are at higher risk of a severe HT on rtPA [3].
Analysis of whether cardiac-emboli were associated with severe HT which was suggested by an autopsy study in which larger confluent haemorrhages were more common in patients with cardio-embolic stroke than in patients with a noncardiac cause of stroke [6].

In cardio-embolic stroke, HT is postulated to occur when the distal migration of embolic fragments allows reperfusion of an ischemic-weakened vascular bed [7]. History of congestive heart failure was associated with an increased risk of $\mathrm{PH}$ and $\mathrm{SICH}$, whereas atrial fibrillation on admission and a history of myocardial infarction were not [3].

Patients with congestive heart failure are at high risk of stroke [8]. A likely mechanism of stroke in these patients is cerebral embolism from a ventricular thrombus [9].

High blood pressure has been related to intracranial haemorrhage after rtPA for ischemic stroke in both experimental and clinical settings $[3,10]$.

In ECASS II study, higher incidence of PH and SICH in rtPAtreated patients who had used aspirin before stroke was in line with the results of the Multicenter Acute Stroke Trial-Italy (MAST-I), in which the effect of the combination of streptokinase and aspirin was assessed [11-14].

In MAST-I, the addition of aspirin to streptokinase significantly increased the number of deaths from intracranial haemorrhages [15].

On the other hand, aspirin was not related to the risk of severe HT either in the ECASS or NINDS trial $[3,15]$.

The determination of risk factors for severe HT on rtPA may help the physician to estimate the risk of thrombolysis and decide whether an individual can be safely treated [3].

In ECASS II, the rates of severe HT on rtPA in patients aged $>70$ years and in those taking aspirin before stroke were very high. Approximately 1 patient of 5 suffered a severe HT in these groups. Certainly, if such high rates were confirmed by an external validation study, thrombolysis should be avoided in these patients [3].

\section{Conclusion}

In our small study group of retrospective data analysis, where we reviewed diagnosis of cardio-embolic stroke, majority of our study patients did not receive rtPA due to timing, risk factors, contraindications or family's refusal for treatment. Our findings raise the possibility of a link between $\mathrm{HT}$ in stroke with $\mathrm{AF}$, especially those with antiplatelet, advancing age and with comorbidities.

The statistical analysis did not support any strong or direct association between the presence of AF and HT. It also failed to confirm any association with co-morbidities and HT. The only association ( $p$-value) were diabetes, advancing age and ischaemic stroke with increased mortality. This may be due to very small sample size. 
Some of the other factors, which can be considered for patients with HT in the absence of AF, are CCF, Intracardiac clots, and Carotid plaques.

Rehabilitation aspect: Some of the patients having HT as a complication of cardio-embolic stroke may need neurosurgical intervention (ICP monitoring, decompressive craniotomy). This further leads to increased morbidity and mortality. The rehabilitation needs of these people are complex with longer length of stay, high dependency level and increased carer burden.

More multidisciplinary rehabilitation resources are needed to manage their complex neurological and medical co-morbidities

We plan to do further study with more sample size along with analyzing carotid Doppler findings.

\section{References}

1. Mattle HP, Schwerzmann M, Seiler Ch. Atrial fibrillation and stroke. Ther Umsch. 2003; 60(9): 527-34.

2. Risk factors for stroke and efficacy of antithrombotic therapy in atria fibrillation: analysis of pooled data from five randomized controlled trials. Arch Intern Med. 1994; 154(13): 1449-57.

3. Vincent Laurre, Rüdiger von Kummer, Achim Müller, Erich Bluhmki. Risk Factors for Severe Hemorrhagic Transformation in Ischemic Stroke Patients Treated With Recombinant Tissue Plasminogen Activator: a Secondary Analysis of the European-Australian Acute Stroke Study (ECASS II). Stroke. 2001; 32(2): 438-41.

4. Wolf PA, Abbott RD, Kannel WB. Atrial fibrillation as an independen risk factor for stroke: the Framingham Study. Stroke. 1991; 22(8): 983-8.

5. Lin HJ, Wolf PA, Kelly-Hayes M, Beiser AS, Kase CS, Benjamin EJ, et al. Stroke Severity in Atrial Fibrillation. The Framingham Study. Stroke. 1996; 27(10): 1760-4.

6. Lodder J, Krijne-Kubat B, Broekman J. Cerebral haemorrhagic infarction at autopsy: cardiac cause and the relationship to the cause of death. Stroke. 1986; 17(4): 626-9.

7. Fisher M, Adams RD. Observations on brain embolism with special reference to the mechanism of haemorrhagic infarction. J Neuropathol Exp Neurol. 1951; 10(1): 92-4.

8. Welch KMA, Louis R Caplan, Donald J Reis, Siesjo Bo J, Weir B Epidemiology and risk factor management. Primer on Cerebrovascular Diseases. San Diego, Calif: Academic Press; 1997: 751-757.

9. FalkRH,Foster E, Coats MH. Ventricular thrombiand thromboembolism in dilated cardiomyopathy: a prospective follow-up study. Am Heart J. 1992; 123(1):136-42.

10. Bowes MP, Zivin JA, Thomas GR, Thibodeaux H, Fagan SC. Acute hypertension but not thrombolysis increases the incidence and severity of hemorrhagic transformation following experimental stroke in rabbits. Exp Neurol. 1996; 141(1): 40-6.

11. Randomized controlled trial of streptokinase, aspirin, and combination of both in treatment of acute ischaemic stroke. Multicentre Acute Stroke Trial-Italy Group. Lancet. 1995; 346(8989): 1509-14.

12. Ciccone A, Motto C, Aritzu E, Piana A, Candelise L. Risk of aspirin use plus thrombolysis after acute ischaemic stroke: a further MAST-I analysis. MAST-I Collaborative Group. Multicentre Acute Stroke Trial-Italy. Lancet. 1998; 352(9131): 880.

13. Levy DE, Brott TG, Haley EC Jr, Marler JR, Sheppard GL, Barsan W, et al. Factors related to intracranial hematoma formation in patients receiving tissue-type plasminogen activator for acute ischemic stroke. Stroke. 1994; 25(2): 291-7.

14. Larrue V, von Kummer R, del Zoppo G, Bluhmki E. Hemorrhagic transformation in acute ischemic stroke: potential contributing factors in the European Cooperative Acute Stroke Study. Stroke. 1997; 28(5): 957-60.

15. The NINDS t-PA Stroke Study Group. Intracerebral hemorrhage after intravenous t-PA therapy for ischemic stroke. Stroke. 1997; 28(11): 2109-18. 